\title{
Pulmonary Function Test
}

National Cancer Institute

\section{Source}

National Cancer Institute. Pulmonary Function Test. NCI Thesaurus. Code C38081.

A broad range of tests that are performed to assess how well lungs inhale and exhale air and how efficiently they transfer oxygen into the blood. 